\title{
In situ Transmission Electron Microscopy Annealing for Crystallization and Phase Stability Studies in the $\mathrm{Ga}_{2} \mathrm{O}_{3}-\mathrm{In}_{2} \mathrm{O}_{3}$ System
}

\author{
Charlotte Wouters ${ }^{1}$, Toni Markurt ${ }^{1}$, Oliver Bierwagen ${ }^{2}$, Christopher Sutton $^{3}$ and Martin Albrecht ${ }^{1}$ \\ 1. Leibniz-Institut für Kristallzüchtung, Berlin, Germany. \\ 2. Paul-Drude-Institut für Festkörperelektronik, Berlin, Germany. \\ 3. Fritz-Haber-Institut der Max-Planck-Gesellschaft, Berlin, Germany. \\ * Corresponding author: charlotte.wouters@ikz-berlin.de
}

The group-III oxides $\mathrm{Ga}_{2} \mathrm{O}_{3}, \mathrm{In}_{2} \mathrm{O}_{3}$, and $\mathrm{Al}_{2} \mathrm{O}_{3}$ and their alloys are wide bandgap materials $(4.8,2.9$, and $8.7 \mathrm{eV}$ ) and belong to the emerging material class of transparent (semi-)conducting oxides (TCO) [1]. In the past years, these TCOs have gained a lot of interest. E.g. the so-called Baliga figure of merit for power electronic devices ranking the materials for such applications is higher for $\mathrm{Ga}_{2} \mathrm{O}_{3}$ than for $\mathrm{SiC}$ and $\mathrm{GaN}$ and field effect transistors made of $\mathrm{Ga}_{2} \mathrm{O}_{3}$ outperform devices based on other materials [1, 2]. Alloying $\mathrm{Ga}_{2} \mathrm{O}_{3}$ with indium can be useful for e.g. solar-blind photo-detectors and transparent Schottky contacts [3]. However, in contrast to other compound semiconductor like the III-nitrides or the IIIarsenides, the constituents of the III-oxide system exhibit different thermodynamically stable crystal structures. While $\mathrm{In}_{2} \mathrm{O}_{3}$ crystallizes in the cubic bixbyite structure, the thermodynamically most favorable phase of $\mathrm{Ga}_{2} \mathrm{O}_{3}$ is the monoclinic $\beta$-phase [6]. For the $\left(\operatorname{In}_{\mathrm{x}} \mathrm{Ga}_{1-\mathrm{x}}\right)_{2} \mathrm{O}_{3}$ system theory predicts the lowest energy structures to be: (i) the monoclinic $\beta$-phase for a indium content lower than $x<0.4$, (ii) a hexagonal phase [4] for a medium indium content of ca. $x=0.5$, and (iii) the cubic bixbyite phase for very high indium contents. However, the stability ranges for all the phases are quite narrow and for wide concentration regions phase separation is expected $[5,6]$. All this makes alloying of III-oxides, which is desired to tailor materials properties for applications, challenging.

To shed more light into this topic and to check the theoretical predictions, we performed in-situ transmission electron microscopy (TEM) annealing experiments of amorphous $\left(\operatorname{In}_{\mathrm{x}} \mathrm{Ga}_{1-\mathrm{x}}\right)_{2} \mathrm{O}_{3}$ thin films deposited on sapphire substrates and studied the crystallization process and stability of the obtained phases. For the in-situ measurements, we used a MEMS chips based environmental TEM holder and heated the samples up to $1000{ }^{\circ} \mathrm{C}$ in different annealing atmospheres at pressures of up to 1 bar. Preliminary investigations showed that special care has to be taken to obtain a clean and damage free electron transparent lamella on the MEMS chip. To avoid specimen artifacts inevitable by FIB lamella lift out preparation approaches (commonly used for in-situ experiments of bulk samples) we developed an alternative preparation method that is based on wedge polishing.

The annealing experiment was first conducted for an amorphous $\mathrm{Ga}_{2} \mathrm{O}_{3}$ thin film in oxygen atmosphere. In this case, part of the material first crystallized in the $\alpha-\mathrm{Ga}_{2} \mathrm{O}_{3}$ phase, which was formed epitaxially on the sapphire, while the rest of the layer transformed to the thermodynamically stable $\beta-\mathrm{Ga}_{2} \mathrm{O}_{3}$ phase. A more interesting result was found for an amorphous $\left(\mathrm{In}_{0.55} \mathrm{Ga}_{0.45}\right)_{2} \mathrm{O}_{3}$ thin film annealed in vacuum. The sample stayed amorphous without any detectable change in the microstructure until $680{ }^{\circ} \mathrm{C}$ was reached. At this temperature first isolated, randomly oriented, crystalline grains were found, apparent as DebyeScherrer rings in the diffraction pattern (Fig. 1). Surprisingly, the crystallites exhibit a cubic structure and the observed diffraction peaks unambiguously fit to the bixbyite phase with an indium content of $x=0.55$. With increasing temperature, the degree of crystallization and size of grains increased. No other phase than the cubic bixbyite structure was found until approximately $900{ }^{\circ} \mathrm{C}$. At higher temperatures, 
we observed degradation of the $\left(\mathrm{In}_{0.55} \mathrm{Ga}_{0.45}\right)_{2} \mathrm{O}_{3}$ cubic phase which separated into structures with different composition. Another $\left(\operatorname{In}_{\mathrm{x}} \mathrm{Ga}_{1-\mathrm{x}}\right)_{2} \mathrm{O}_{3}$ sample with an even lower indium content of $\mathrm{x}=0.33$ showed similar results. The amorphous material crystallized again as a cubic bixbyite phase. Annealing experiments performed in oxygen and vacuum revealed qualitatively the same results, though crystallization in oxygen is initiated at $\approx 100 \mathrm{~K}$ higher temperature. This could probably be attributed to enhanced local atomic movement in the presence of oxygen vacancies for vacuum annealing.

Summarizing our results, we showed that in the $\mathrm{In}_{2} \mathrm{O}_{3}-\mathrm{Ga}_{2} \mathrm{O}_{3}$ system a metastable phase can be stabilized if crystallization is initiated from an amorphous film. This finding is not only remarkable in general for fundamental physics, but for the present example this is also from application point of view interesting since the accessible composition range of alloys is extended. The appearance of the metastable cubic phase can be explained by the specific crystallization pathway. The chemical configuration as well as the next-nearest-neighbor bonding coordination of the ensemble of atoms is predefined by their status in the amorphous matrix. When a crystallite is formed, there is a large kinetic barrier to break bonds necessary to swap atoms to reach the thermodynamic ground state. Instead, the crystallized phase will be one of high entropy, with indium and gallium randomly distributed on the lattice sites. We can describe this as an effective high temperature state, for which the formation energy of the cubic phase becomes lower in energy than that of the monoclinic phase (Fig.2). This results from the presence of only one type of coordination site (six-fold) for the cations in the cubic phase, in contrast to the monoclinic (and hexagonal) phases. Due to the site-preference of indium for this six-fold coordination, the cubic phase is therefore most suited to accommodate this high amount of configurational entropy imposed by the amorphous phase. At higher annealing temperatures, the metastable material will eventually then become as in our experiment unstable and decompose.

References:

[1] Higashiwaki et al., Appl. Phys. Lett. 100 (2012), p. 013504.

[2] Chabak et al., Appl. Phys. Lett. 109 (2016), p. 213501.

[3] Wenckstern et al., Semicond. Sci. Technol. 30 (2015), p. 024005.

[4] Shannon and Prewitt, J. inorg. nucl. Chem. 30 (1968), p. 1389.

[5] Peelaers et al., Phys Rev B 92 (2015), p. 085206.

[6] Maccioni et al., Appl. Phys. Express 9 (2016), p. 041102.

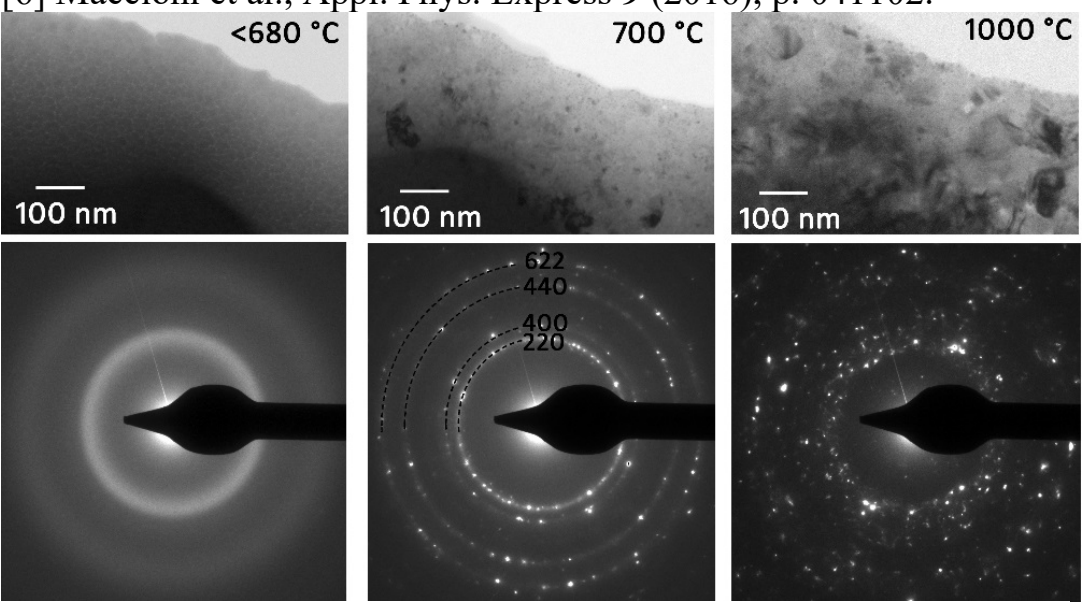

Figure 1. Vacuum annealing of a $\left(\operatorname{In}_{0.55} \mathrm{Ga}_{0.45}\right)_{2} \mathrm{O}_{3}$ thin film. Top row: Bright field TEM, Bottom row: corresponding diffraction pattern with (middle) Debye-Scherrer rings for bixbyite $\left(\mathrm{In}_{0.55} \mathrm{Ga}_{0.45}\right)_{2} \mathrm{O}_{3}$.

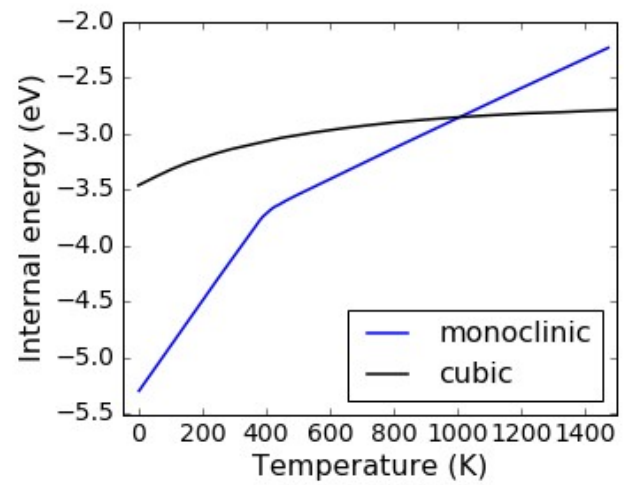

Figure 2. Calculated internal energy as a function of temperature for the monoclinic and cubic $\left(\operatorname{In}_{\mathrm{x}} \mathrm{Ga}_{1-\mathrm{x}}\right)_{2} \mathrm{O}_{3}$ alloy at $\mathrm{x}=0.5$. 